\title{
How accurately can we age-date solar-type dwarfs using activity/rotation diagnostics?
}

\author{
Eric E. Mamajek \\ Department of Physics \& Astronomy, \\ University of Rochester, Rochester, NY 14624 USA \\ email: emamajek@pas.rochester.edu
}

\begin{abstract}
It is well established that activity and rotation diminishes during the life of sun-like main sequence $(\sim \mathrm{F} 7-\mathrm{K} 2 \mathrm{~V})$ stars. Indeed, the evolution of rotation and activity among these stars appears to be so deterministic that their rotation/activity diagnostics are often utilized as estimators of stellar age. A primary motivation for the recent interest in improving the ages of solar-type field dwarfs is in understanding the evolution of debris disks and planetary systems. Reliable isochronal age-dating for field, solar-type main sequence stars is very difficult given the observational uncertainties and multi-Gyr timescales for significant structural evolution. Observationally, significant databases of activity/rotation diagnostics exist for field solar-type field dwarfs (mainly from chromospheric and X-ray activity surveys). But how well can we empirically age-date solar-type field stars using activity/rotation diagnostics? Here I summarize some recent results for F7-K2 dwarfs from an analysis by Mamajek \& Hillenbrand (2008), including an improved "gyrochronology" [Period(color, age)] calibration, improved chromospheric $\left(R_{\mathrm{HK}}^{\prime}\right)$ and $\mathrm{X}$-ray $\left(\log \left(L_{\mathrm{X}} / L_{b o l}\right)\right)$ activity vs. rotation (via Rossby number) relations, and a chromospheric vs. X-ray activity relation that spans four orders of magnitude in $\log \left(L_{\mathrm{X}} / L_{b o l}\right)$. Combining these relations, one can produce predicted chromospheric and X-ray activity isochrones as a function of color and age for solar type dwarfs.
\end{abstract}

Keywords. Sun: (activity, rotation), stars: (activity, chromospheres, coronae, fundamental parameters, late-type, low-mass, rotation), Galaxy: evolution

\section{Introduction}

Observational and theoretical studies regarding the evolution of circumstellar disks and planetary systems have fueled a renewed interest in assessing how accurately we can determine the ages for solar-type field dwarfs (Mamajek et al. 2008, ; Meyer, this volume). For field stars we can place reasonable constraints on their effective temperatures, luminosities, and metallicities from spectroscopic, photometric, and astrometric measurements. Plotting these observables against theoretical evolutionary tracks allows us to infer ages and masses. However, for main sequence solar-type stars, the observational uncertainties in a star's HRD diagram position can be large enough that they encompass a large fraction of the star's main sequence lifetime, even for stars with precise distances and metallicities (e.g. Nordström et al. 2004; Valenti \& Fischer 2005; Takeda et al. 2007). The situation is worse for the hordes of stars lacking trigonometric parallaxes and metallicity estimates. For this reason, we are motivated to explore alternative age indicators beyond deriving individual isochronal ages.

It has been long appreciated that solar-type stars lose angular momentum via a magnetized wind, spin down, and become less active during their main sequence phase (e.g. Skumanich 1972; Soderblom et al. 1991). Here I discuss recent efforts by the author and collaborators to improve the estimation of ages for solar-type $(\sim F 7-K 2)$ field dwarfs using rotation/activity diagnostics. By "activity diagnostics", I will discuss two common 
examples: the Ca II $\mathrm{H} \& \mathrm{~K}$ chromospheric activity index $\log R_{\mathrm{HK}}^{\prime}$, and the X-ray-tobolometric luminosity ratio $\log \left(L_{\mathrm{X}} / L_{b o l}\right)\left(=\log R_{\mathrm{X}}\right)$ in the $0.2-2.4 \mathrm{keV}$ band (ROSAT band). For more exhaustive and wavelength-balanced reviews, especially from the perspective of the Sun's evolution, I refer the reader to Güdel (2007), Ayres (1997), and Walter \& Barry (1991).

For the Sun, radiometric dating of the oldest meteorites have converged on an age within a few Myr of 4.57 Gyr (e.g. Baker et al. 2005). Pleasingly, solar models which match the observed helioseismological constraints (sound speed profiles, acoustic modes) can produce the Sun with an age within a few percent of the meteoritic age (Houdek \& Gough 2008). For members of nearby young open clusters (e.g. the Pleiades, Hyades, etc.), detailed modelling of the HR diagram positions of the high-mass members, and HRD positions and Li-depletion pattern of the low-mass members, has led to age-dating with claimed accuracy of $\sim 5-15 \%$ (e.g. de Bruijne et al. 2001; Barrado y Navascués, et al. 2004). Recent results for the small sample of solar-type stars which have been asteroseismologically observed and modeled are also yielding age uncertainties of typically $\sim 5-15 \%$ (Thévenin et al. 2002; Eggenberger et al. 2004), with some claims of even $\sim 1 \%$ (Mosser et al. 2008). While the observational data for these well-studied clusters and asteroseismological target stars is impeccable, the accuracy of the inferred cluster ages hinge on the input physics (e.g. treatment of convection, opacities, etc.) and abundances of the stellar evolution models, both of which are intimately tied to solar modeling efforts. While the Sun provides us with a "gold" age standard (< few \% accuracy) and open clusters and asteroseismological targets provide us "silver" age standards $(\sim 15 \%)$, how well can we estimate ages for solar-type field dwarfs using "bronze" indicators like rotation and activity?

\section{Ages from activity and/or rotation}

\subsection{The chromospheric activity-age correlation}

In solar-type stars, the majority of chromospheric and X-ray activity is believed to be generated as a result of the stellar magnetic dynamo. The strength of the dynamo and its ability to nonthermally heat the outer atmospheres of Sun-like stars is ultimately tied to stellar rotation - and more specifically - differential rotation (Noyes et al. 1984; Donahue et al. 1996). Both activity and rotation among Sun-like stars are observed to decay with isochronal age (e.g. Wilson 1963; Skumanich 1972; Soderblom et al. 1991). As an illustration of this, in Fig. 1 (left) we plot chromospheric activity $\log R_{\mathrm{HK}}^{\prime}$ vs. color for the Sun and members of age-dated clusters (Mamajek \& Hillenbrand 2008). Using our large modern database of activity and age estimates, we find shortcomings among all previous activity-age relations. The new fit to the cluster and field star activity-age data is shown as a solid line in Fig. 1 (right):

$$
\log \tau=-38.053-17.912 \log R_{H K}^{\prime}-1.6675 \log \left(R_{H K}^{\prime}\right)^{2}
$$

As is obvious from the leftside figure of Fig. 1, there are color-dependent effects which force us to dismiss a simple activity-age relationship as an oversimplification. Simply using this activity-age polynomial can provide age estimates of $\sim \pm 0.25$ dex or $60 \%$ accuracy ( $1 \sigma$; uncertainties come from investigating the scatter in inferred ages among coeval cluster or binary samples), however color-dependent systematic effects will be present. Mamajek \& Hillenbrand (2008) were unable to find a simple way to parameterize age as a function of activity and color which simultaneously satisfied the available cluster, binary, 

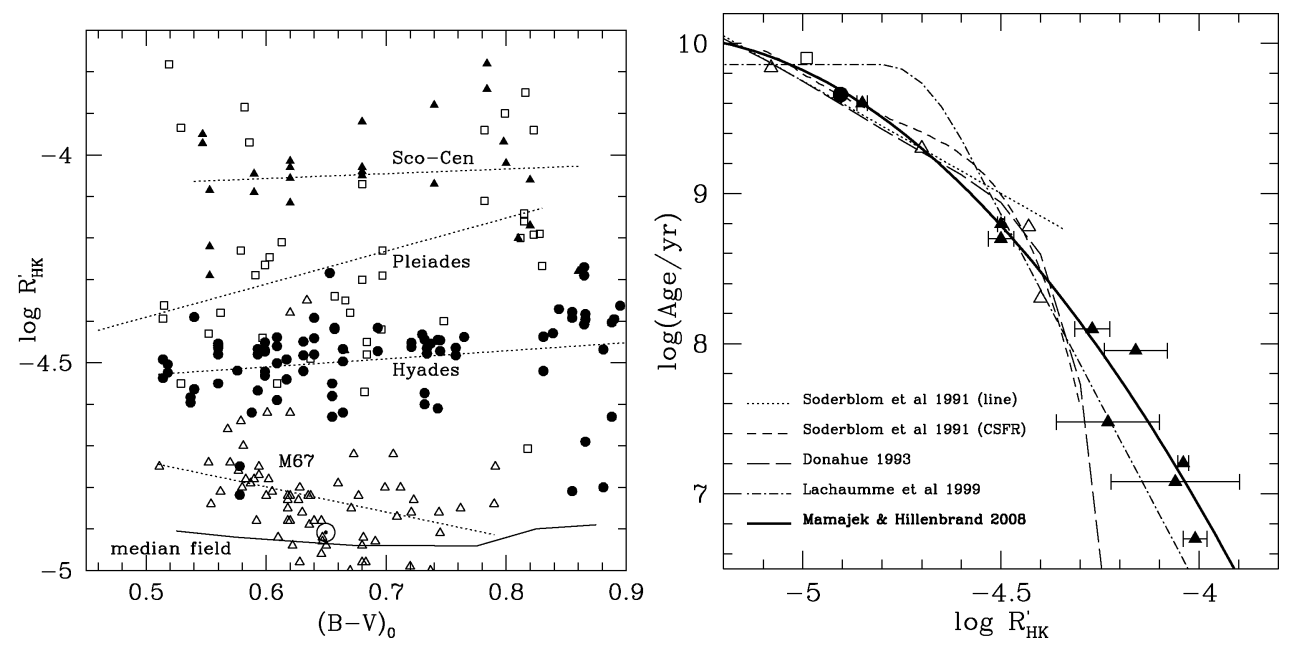

Figure 1. Left: Intrinsic B-V color vs. chromospheric activity index $\log R_{\mathrm{HK}}^{\prime}$ for members of age-dated clusters and the Sun (from Mamajek \& Hillenbrand 2008). Sco-Cen members are typically $\sim 5-17 \mathrm{Myr}$ old (filled triangles), the Pleiades are $\sim 130 \mathrm{Myr}$ old (open squares), the Hyades are $\sim 625$ Myr old (filled circles), and M67 is $\sim 4$ Gyr old (open triangles; see Mamajek \& Hillenbrand 2008, and references therein). The median $\log R_{\mathrm{HK}}^{\prime}$ value for field dwarfs as a function of $(B-V)_{0}$ is plotted. Right: Color-independent activity-age relationships from previous studies and Mamajek \& Hillenbrand (2008). The best fit to the cluster data and a sample of isochronally-dated older field dwarfs is $\log (\tau / \mathrm{yr})=-38.053-17.912 \log R_{\mathrm{HK}}^{\prime}-1.6675 \log R_{\mathrm{HK}}^{\prime}{ }^{2}$ (solid line). However cluster and binary $\log R_{\mathrm{HK}}^{\prime}$ data suggests that one needs to allow for color-dependence. This is best taken into account by converting $\log R_{\mathrm{HK}}^{\prime} \rightarrow$ rotation $\rightarrow$ age via the Rossby number and a gyrochronology relation.

and field star datasets. But there is hope in the form of the activity-rotation correlation (e.g. Noyes et al. 1984) and the gyrochronology relations (Barnes 2007).

\subsection{Ages from chromospheric activity via rotation}

Theoretical models exist for explaining the decay of rotation speeds among solar-type stars due to angular momentum loss via magnetized winds and changes in the moment of inertia of the star (Kawaler 1988). Some of the model parameters are poorly constrained, (e.g. mass loss, magnetic field geometry), but large rotation period datasets for clusters can be used to constrain the parameters (Irwin, this volume). Empirically, however, one can fit a series of simple curves in color-period-age space. These "gyrochronology" curves introduced by Barnes (2007) and improved upon by Mamajek \& Hillenbrand (2008) can be used to derive ages from rotation rates with statistical accuracy of order $\sim 15 \%$. For the solar-type dwarfs, Mamajek \& Hillenbrand (2008) fit a gyrochronology relation for period $P$ in days and age $t$ in Myr:

$$
P(B-V, t)=(0.407 \pm 0.021)\left[(B-V)_{o}-0.495 \pm 0.010\right]^{0.325 \pm 0.024}(t / \mathrm{Myr})^{0.566 \pm 0.008}
$$

Rotation rate can be tied to dynamo strength via the Rossby number $\left(R_{o}\right)$, which is observationally defined as the rotation period divided by an estimate of the local convective turnover time just above the convective-radiative boundary $\left(\tau_{c}\right.$; e.g. Noyes et al. 1984). Using the best available data for solar-type dwarfs, Mamajek \& Hillenbrand (2008) find the strong correlation between the Rossby number and chromospheric activity for "normal" and "inactive" $\sim \mathrm{F} 7-\mathrm{K} 2$ dwarfs $\left(\log R_{\mathrm{HK}}^{\prime}<-4.35\right)$ to be:

$$
\log R_{H K}^{\prime}=-4.522-0.337\left(R_{o}-0.814\right)
$$


Through applying the conversion activity $\rightarrow$ rotation $\rightarrow$ age via the Rossby number and gyrochronology, an analysis of (presumably) coeval stars in resolved binaries and star clusters suggests that the derived ages have precision of $\sim \pm 0.1-0.2 \operatorname{dex}(\sim 25-50 \% ; 1 \sigma)$. By combining a gyrochronology relation with the $\log R_{\mathrm{HK}}^{\prime}$-Rossby number correlation, one can predict chromospheric activity as a function of color and age for solar-type dwarfs ("gyrochromochrones"; Fig. 2). When combining the activity vs. rotation and rotation vs. age relations, it becomes apparent that for a given chromospheric activity level $\log R_{\mathrm{HK}}^{\prime}$, the late F-type and early G-type stars are systematically younger than the late G-type and early K-type stars. In the future, it will be prudent to take into account the effects of metallicity in Fig. 2, and produce isochrones in mass-metallicity-activity.

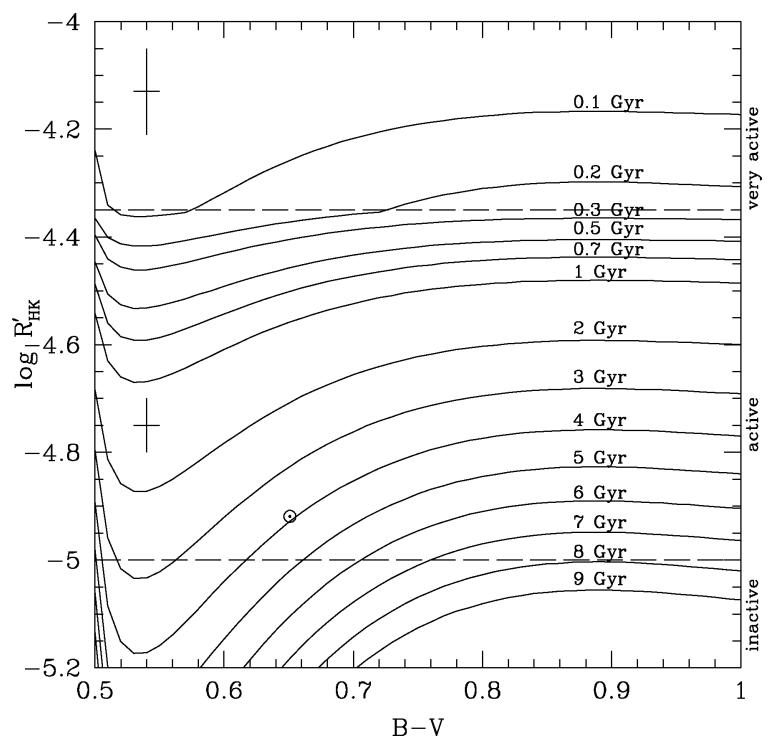

Figure 2. Predicted chromospheric activity levels as a function of age ("gyrochromochrones"), from combining the age-rotation relations (Eqn. 2.2) with the rotation-activity relations (Eqn. 2.3 for age $>100 \mathrm{Myr}$ ). Typical uncertainty bars are shown in the very active and active regimes, reflecting the r.m.s. in the Rossby number-activity fits, and typical photometric errors. The behavior of the gyrochromochrones at the blue end (i.e. the obvious upturn) is not well-constrained, and is particularly sensitive to the $c$ parameter in the gyrochronology fits. Figure from Mamajek \& Hillenbrand (2008).

\subsection{Coronal $X$-rays as an age indicator}

It is clear that one can estimate useful stellar ages for solar-type dwarfs from rotation periods (e.g. Barnes 2007), however rotation periods are currently difficult to measure in large numbers for all but the most active, starspotted stars (although periods can be inferred from long-term monitoring of chromospheric emission; Donahue et al. 1996). For these older stars lacking rotation periods, of order $\sim 10^{3.5}$ solar-type field dwarfs have chromospheric activity measurements (predominantly from large surveys by e.g. Henry et al. 1996; Wright et al. 2004; Gray et al. 2006) to help us estimate their ages. However, another larger, and mostly untapped, stellar activity database exists for age estimation: $\mathrm{X}$-ray fluxes.

Using ROSAT soft X-ray (0.2-2.4 keV) fluxes, Sterzik \& Schmitt (1997) demonstrated that coronal X-ray activity $\left(\log \left(L_{\mathrm{X}} / L_{b o l}\right)=\log R_{\mathrm{X}}\right)$ scales with chromospheric activity $\log R_{\mathrm{HK}}^{\prime}$ over $\sim 4$ orders of magnitude in $\log R_{\mathrm{X}}$ and $\sim 1$ order of magnitude in $\log R_{\mathrm{HK}}^{\prime}$. 
Mamajek \& Hillenbrand (2008) improved the correlation through including more highand low-activity stars, and provided an improved quantification of this correlation for solar-type dwarfs:

$$
\log R_{H K}^{\prime}=(-4.54 \pm 0.01)+(0.289 \pm 0.015)\left(\log R_{X}+4.92\right)
$$

with an r.m.s. scatter of 0.06 in $\log R_{\mathrm{HK}}^{\prime}$. The inverse relation is:

$$
\log R_{X}=(-4.90 \pm 0.04)+(3.46 \pm 0.18)\left(\log R_{H K}^{\prime}+4.53\right)
$$

with an r.m.s. of $0.19 \operatorname{dex}(\sim 55 \%)$ in $\log R_{\mathrm{X}}$. Equation 2.5 is statistically consistent with the relation found by Sterzik \& Schmitt (1997), but our uncertainties are $\sim 2 \times$ smaller.

As with the chromospheric activity, their is a strong correlation between coronal X-ray activity and rotation via the Rossby number:

$$
\mathrm{R}_{\mathrm{o}}=(0.86 \pm 0.02)-(0.79 \pm 0.05)\left(\log R_{X}+4.83\right)
$$

This best fit appears to be useful over $\sim 4$ orders of magnitude in $\log R_{\mathrm{X}} \quad\left(-7<\log R_{\mathrm{X}}\right.$ $<-4)$. The fit is similar to the linear-log fit quoted by Hempelmann et al. (1995), but is severely at odds with the oft-cited $\log R_{\mathrm{X}}$ vs. $\log R_{o}$ fit quoted by Randich et al. (1996). Hence, one can relate X-ray fluxes to rotation periods via the Rossby number (typically $\sim 0.251 \sigma$ accuracy in $R_{o}$ ), and estimate ages from the periods via a gyrochronology relation. The typical spread in $\log R_{\mathrm{X}}$ as a function of age is $\pm 0.4 \operatorname{dex}(1 \sigma)$ and should be factored into the age uncertainty. It appears that a few hundred second X-ray snapshot with an X-ray satellite can be used to predict the multi-decadal average value of $\log R_{\mathrm{HK}}^{\prime}$ to within $\pm 0.1(1 \sigma)$ accuracy (minimum age precision $\sim 30-50 \%$ ). A star with X-ray emission similar to that of the Sun can be seen out to $\sim 15 \mathrm{pc}$ in the ROSAT All-Sky Survey, and younger, more active stars can be seen to larger distances. Using X-ray emission measured by the ROSAT All-Sky Survey, one should (in principle) be able to derive useful ages for $\sim 10^{2-3}$ solar-type field dwarfs in the solar neighborhood.

The very active stars $\left(\log R_{\mathrm{HK}}^{\prime}>-4.35 ; \log R_{\mathrm{X}}>-4.0\right)$ have negligible correlation between rotation and activity (i.e. the "saturated" regime). So while setting upper limits to ages might be fruitful in the saturated regime, quoting exact ages appears not to be.

\section{Implications for nearby Sun-like field dwarfs}

What we have constructed are useful empirical relations between stellar measurements, which when combined, yield a parameter more difficult to measure: age. In future work, we would like to understand the physics underlying these empirical relations in terms of how it constrains stellar dynamo theory (e.g. Montesinos et al. 2001) and the evolution of stellar angular momentum (e.g. Kawaler 1988). For the time being, let us use our new and improved rotation-activity-age tools to see what their implications are.

We have already seen that the evolution of activity appears to be fairly color/massdependent among solar-type dwarfs (Fig. 2), contrary to previous studies which employed a color/mass-independent activity-age correlation. As a first use of our activity $\rightarrow$ rotation $\rightarrow$ age calibrations, we constructed a histogram of the chromospheric activity-derived ages for a volume-limited $(d<16 \mathrm{pc})$ sample of the nearest 108 solar-type dwarfs to the Sun†. A table of the names, parallaxes, B-V colors, $\log R_{\mathrm{HK}}^{\prime}$ values, absolute magnitudes, spectral types, and inferred ages for the sample stars is given in Table 13 of Mamajek

$\dagger$ The ages for these nearest solar-type dwarfs will be of astrobiological interest for proposed missions designed to image and take spectra of extrasolar terrestrial planets, like the TPF and Darwin (Kaltenegger et al. 2007) and New Worlds Observer (Cash et al. 2005) 

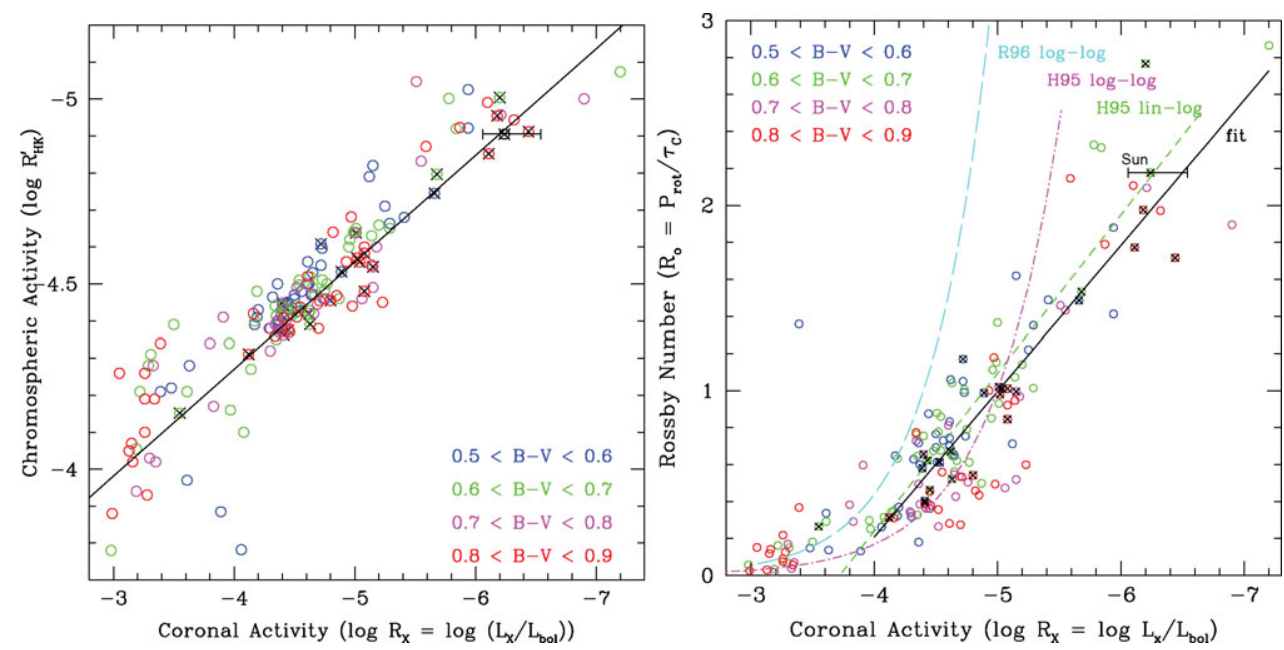

Figure 3. Left: $\log R_{\mathrm{X}}$ vs. $\log R_{\mathrm{HK}}^{\prime}$ for solar-type dwarfs with known rotation periods and chromospheric and X-ray activity levels (from Mamajek \& Hillenbrand 2008). Stars from Donahue et al. (1996) and Baliunas et al. (1996) with well-determined periods also have dark Xs and conveniently provide an X-ray unbiased sample via the ROSAT All-Sky Survey. Shaded color bins are illustrated in the legend. The Solar X-ray and $\log R_{\mathrm{HK}}^{\prime}$ datum is described in Mamajek \& Hillenbrand (2008). Right: $\log R_{\mathrm{X}}$ vs. Rossby number $R_{o}$ for stars in our sample of solar-type stars with known rotation periods and chromospheric and X-ray activity levels (from Mamajek \& Hillenbrand 2008). Donahue-Baliunas stars with well-determined periods also have dark Xs. Previously published $R_{\mathrm{X}}$ vs. $R_{o}$ fits are drawn: cyan long-dashed line is a log-log fit from Randich et al. (1996), magenta dot-dashed line is a log-log fit from Hempelmann et al. (1995), and the green dashed line is a linear-log fit from Hempelmann et al. (1995). Our new log-linear fit for stars in the range $-7<\log R_{\mathrm{X}}<-4$ is the solid dark line, consistent with the Hempelmann linear-log relation. Saturated X-ray emission $\left(\log R_{\mathrm{X}}>-4\right)$ is consistent with $R_{o}<0.5$.

\& Hillenbrand (2008). We plot the fruits of this effort as a histogram of inferred ages in Figure 4. The histogram can not be directly interpreted as a "star-formation history" at this time, as we have not accounted for the effects of kinematic disk-heating, the loss of some older stars due to stellar evolution (given the constraint that the "dwarf" stars must lie within 1 mag of the main sequence), and the effects of metallicity on the sample. As these effects will mostly conspire to skew our conclusions regarding the old end of the histogram (i.e. evolved and/or metal-poor and/or high-velocity stars), we focus on the stars younger than the Sun. First, we note that when a simple activity $\rightarrow$ age relation is adopted, one sees a pronounced dip in the age histogram at age $\sim 2-3$ Gyr, right in the region of the "Vaughan-Preston gap" (Vaughan \& Preston 1980). One one derives age using the recommended activity $\rightarrow$ rotation $\rightarrow$ age (via the Rossby number and revised gyrochronology relations), one gets a much flatter age distribution between 0-6 Gyr. As our color-magnitude selection biases should have negligible impact on the age distribution of these young to middle-aged dwarfs, it appears that the histogram is consistent with a more-or-less flat star-formation history over the past $\sim 5$ Gyr or so. The histogram is in disagreement with assertions in previous historical studies (e.g. Barry 1988, which used an activity-age relation) which concluded that there has been a recent enhancement of the stellar birth-rate in the past $\sim$ Gyr, which followed a lower birth-rate 2-3 Gyr ago. Applying our age-estimation methods to a larger sample of the nearest solar-type stars out to $\sim 25-40 \mathrm{pc}$ should place our conclusions on firmer statistical footing. 


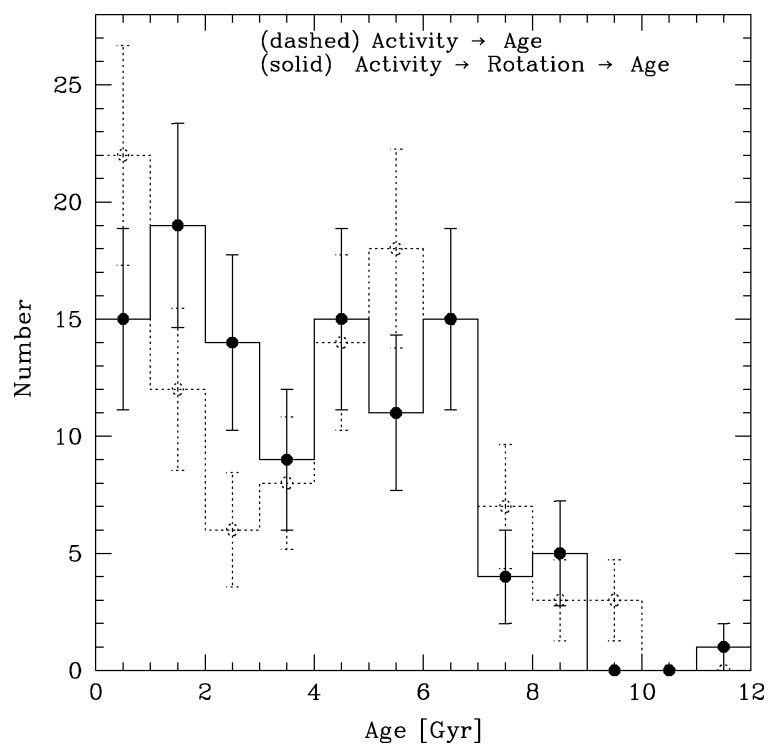

Figure 4. Histogram of inferred ages for the nearest 108 solar-type dwarfs (F7-K2V) within 16 pc (from Mamajek \& Hillenbrand 2008). Dashed histogram is for ages inferred directly from chromospheric activity using equation 3. Solid histogram is for ages derived from converting activity $\log R_{\mathrm{HK}}^{\prime}$ to rotation period, then converting rotation period and color to age via the Rossby number and revised gyro relation.

These techniques should also be prove valuable for more accurately assessing the ages of extrasolar planetary systems (Mamajek \& Slipski, in prep.) and dusty debris disk systems (Hillenbrand et al., in prep.).

\section{Acknowledgements}

The author collaborated with Lynne Hillenbrand on much of the material presented for this talk (presented in Mamajek \& Hillenbrand 2008), and acknowledges helpful conversations with D. Soderblom, J. Stauffer, and M. R. Meyer.

\section{References}

Ayres, T. R. 1997, Jnl. Geophys. Res., 102, 1641

Baker, J., Bizzarro, M., Wittig, N., Connelly, J., \& Haack, H. 2005, Nature, 436, 1127

Baliunas, S., Sokoloff, D., \& Soon, W. 1996, ApJ, 457, L99

Barnes, S. A. 2007, ApJ, 669, 1167

Barrado y Navascués, D., Stauffer, J. R., \& Jayawardhana, R. 2004, ApJ, 614, 386

Barry, D. C. 1988, ApJ, 334, 436

de Bruijne, J. H. J., Hoogerwerf, R., \& de Zeeuw, P. T. 2001, A\&A, 367, 111

Cash, W., Kasdin, J., Seager, S., \& Arenberg, J. 2005, Proc. SPIE, 5899, 274

Donahue, R. A., Saar, S. H., \& Baliunas, S. L. 1996, ApJ, 466, 384

Eggenberger, P., et al. 2004, A\&A, 417, 235

Gray, R. O., et al. 2006, AJ, 132, 161

Güdel, M. 2007, Living Reviews in Solar Physics, 4, 3

Hempelmann, A., et al. 1995, A\&A, 294, 515

Henry, T. J., Soderblom, D. R., Donahue, R. A., \& Baliunas, S. L. 1996, AJ, 111, 439

Houdek, G. \& Gough, D. O. 2008, IAU Symposium, 252, 149

Kaltenegger, L., Traub, W. A., \& Jucks, K. W. 2007, ApJ, 658, 598

Kawaler, S. D. 1988, ApJ, 333, 236 
Mamajek, E. E., Barrado y Navascués, D., Randich, S., Jensen, E. L. N., Young, P. A., Miglio, A., \& Barnes, S. A. 2008, 14th Cambridge Workshop on Cool Stars, Stellar Systems, and the Sun, 384, 374

Mamajek, E. E. \& Hillenbrand, L. A. 2008, ApJ, 687, 1264

Montesinos, B., Thomas, J. H., Ventura, P., \& Mazzitelli, I. 2001, MNRAS, 326, 877

Mosser, B., et al. 2008, A\&A, 488, 635

Nordström, B., et al. 2004, A\&A, 418, 989

Noyes, R. W., et al. 1984, ApJ, 279, 763

Randich, S., Schmitt, J. H. M. M., Prosser, C. F., \& Stauffer, J. R. 1996, A\&A, 305, 785

Skumanich, A. 1972, ApJ, 171, 565

Soderblom, D. R., Duncan, D. K., \& Johnson, D. R. H. 1991, ApJ, 375, 722

Sterzik, M. F. \& Schmitt, J. H. M. M. 1997, AJ, 114, 1673

Takeda, G., et al. 2007, ApJS, 168, 297

Thévenin, F., et al. 2002, A\&A, 392, L9

Valenti, J. A. \& Fischer, D. A. 2005, ApJS, 159, 141 (VF05)

Vaughan, A. H. \& Preston, G. W. 1980, PASP, 92, 385

Walter, F. M. \& Barry, D. C. 1991, The Sun in Time, 633

Wilson, O. C. 1963, ApJ, 138, 832

Wright, J. T., Marcy, G. W., Butler, R. P., \& Vogt, S. S. 2004, ApJS, 152, 261

\section{Discussion}

D. Soderblom: First, maybe it's a coincidence, but the line for Rossby number $=1$ runs right down the middle of the "Vaughan-Preston gap," and that may explain the build-up of stars at $\log R_{\mathrm{HK}}^{\prime}=-4.5$. Second, have you compared activity ages to isochrones ages? When I do I see zero correlation.

E. Mamajek: Rossby number $=1$ depends on the choice of convective overturn time, which differs by a factor of a few among the models. I adopted those from Noyes et al. (1984). The scatter between ages from activity or gyrochronology and those from isochrones (Valenti \& Fischer 2005) is large; however, Valenti has suggested that Takeda et al. (2007) ages are to be preferred, but I have not yet compared to those.

F. WALter: The Rossby number is a convenient way to sweep a lot of our ignorance about convection under the rug (or at least into a single parameter). How well do we really understand convective turnover timescales in convective stars, and how might this uncertainty affect the details of your activity-age relations?

E. MAMAJEK: Modelers have evaluated convective turnover times differently - primarily at different depths with respect to the base of the convection zone - but there appears to broad agreement in the relative turnover times for main sequence stars as a function of mass. Combining the theoretical turnover times with the observed rotation and activity data shows that a strong correlation between Rossby number and activity exists over a wide range of parameter space for solar-type dwarfs when one uses MLT models with $\alpha=1.9$ (see Noyes et al. 1984 and Montesinos et al. 2001). Deriving rough ages for solartype dwarfs using activity is then supported by two empirical correlations: the activityrotation relation (via the Rossby number) and the rotation-age relation (gyrochronology; see talk by S. Barnes). 